$\mathrm{kDa}$ VEGF protein (a monomeric VEGF-164 in reducing condition) decreased 14 days after cysteamine, and increased in groups with i.d. injection of either ND of VEGF, AV of VEGF or PDGF 7 days after cysteamine. In contrast, i.v. injection of AV of both VEGF and PDGF diminished $23 \mathrm{kDa}$ VEGF 7 days after cysteamine, but increased it in 14 days. Conclusions: 1) Gene therapy with naked DNA of VEGF, adenovirus of VEGF or PDGF significantly accelerated chronic duodenal ulcer healing in 14 but not 7 days after the administration of duodenal ulcerogen cysteamine. 2) Increased levels of VEGF were detected by Western blotting in duodenal mucosa after both VEGF and PDGF gene therapy. 3) Thus, VEGF and PDGF gene therapy seems to be a new option to achieve a rapid duodenal ulcer healing.

Hwang, Ms. Sue

\section{A New Class of Polymers for the Delivery of Macromolecular Therapeutics}

\author{
Sue Hwang \\ Caltech, Chemical Engineering, Mail Code 210-4, Pasadena, CA 91125, \\ USA
}

Cationic polymers show promise for the in vitro and in vivo delivery of macromolecular therapeutics. Known cationic polymers, e.g., poly-(L)lysine (PLL) and polyethylenimine (PEI), have been employed in native and modified forms for the delivery of plasmid DNA (pDNA) and reveal varying levels of toxicity. Here, we report the preparation of a new class of cationic polymers that are specifically designed to deliver macromolecular therapeutics. Linear, cationic, $\beta$-cyclodextrin $(\beta-C D)$-containing polymers (CD-polymers) are synthesized by copolymerizing difunctionalized $\beta-\mathrm{CD}$ monomers (AA) with other difunctionalized comonomers (BB) such that an AABBAABB product is formed. The $\beta$-CD polymers are able to bind $\sim 5 \mathrm{kbp}$ pDNA above polymer to DNA (+/-) charge ratios of 1.5 , compact the bound pDNA into particles of approximately $100-150 \mathrm{~nm}$ in size at charge ratios above 5+/-, and transfect cultured cells at charge ratios above 10+/-. In vitro transfections with the new $\beta$-CD-polymers are comparable to the best results obtained in our hands with PEI and Lipofectamine. Some cell line-dependent toxicities are observed for serum-free transfections; however, no toxicity is revealed at charge ratios as high as $70+/$ - in transfections conducted in 10\% serum. Single IV and IP doses as high as 200 $\mathrm{mg} / \mathrm{kg}$ in mice showed no mortalities.
Kingsman, Susan

\section{Preclinical Evaluation of Human Cytochrome P450 2 b6 (Cyp2b6) as a Therapeutic Gene Delivered by a Retroviral Vehicle for Gene-Directed Enzyme Prodrug Therapy (GDEPT) of Cancer}

\author{
S.M. Kingsman, O. Kan, L. Griffiths, S. Iqball, D. \\ Baban, M. Uden, H. Spearman, A. Slade, A.J. \\ Kingsman \& S. Naylor
}

Oxford BioMedica (UK) Ltd, Medawar Centre, Oxford Science Park, Oxford, OX4 4GA, UK

We are currently interested in developing gene therapy for the eradication of solid tumours using GDEPT approaches. The therapeutic gene employed in our company is human cytochrome P450 2B6 (CYP2B6) which encodes a prodrug metabolising enzyme. Cyclophosphamide is one such prodrug and the active metabolite phosphamide mustard subsequently produced crosslinks DNA causing DNA damage and subsequent cell death. A novel retroviral vector encoding P450 2B6 (MetXia-P450) was constructed and used to transduce human tumour cell lines HT29 and T47D. MetXia-P450 transduction sensitises these cells to the anti-proliferative or cytotoxic effect of the prodrug cyclophosphamide. The results from a range of biochemical and cellular biological assays demonstrate inhibition on cell cycle progression and DNA synthesis and a reduction in clonogenic survival in cells transduced with MetXia$\mathrm{P} 450$, in response to cyclophosphamide. Cytotoxic activity was accompanied by a significant bystander effect that was even more evident in 3-dimensional multicellular spheroid models than monolayers suggesting that this may be a more appropriate model for assessing the GDEPT approach. We have applied this approach in an active gene therapy setting using established subcutaneous tumour xenografts. Here we show for the first time the efficacy of P450-based GDEPT strategy mediated by direct intratumoral injection of the therapeutic retroviral vector. MetXia-P450 is being evaluated in phase I/II clinical trials in breast and ovarian cancer. 